Message Framing Influences Perceptions of Feedback (In)directness

Franki Y. H. Kung ${ }^{1} \quad$ Abigail A. Scholer ${ }^{2}$

${ }^{1}$ Department of Psychological Sciences, Purdue University

${ }^{2}$ Department of Psychology, University of Waterloo

Correspondence concerning this manuscript should be addressed to Franki Kung, Department of Psychological Sciences, Purdue University, 703 Third Street, West Lafayette, IN 47907, USA; E-mail: $\underline{\text { rankikung@purdue.edu }}$

Funding

This work was supported by the Social Sciences and Humanities Research Council of Canada though a grant (Grant \#435-2017-0184) awarded to A.S. and Vanier Scholarship (CGV-SSHRC00379) awarded to F.K.

Acknowledgement

We thank Richard Eibach for his helpful direct feedback on an earlier version of the manuscript and Maria Bizev, Anna Chang, and Kailey Dudek for their assistance in data collection and coding written feedback.

Note. This pre-print is the authors' final copy before publication, but may not exactly replicate the final published version. It is not the copy of record. 


\begin{abstract}
Communication varies in indirectness, influencing the effectiveness of the message as well as interpersonal dynamics. However, this issue was not studied empirically in the feedback literature. Integrating communication indirectness and message framing theories, we propose that whether success and failure feedback are framed as a negation (non-losses, non-gains) or affirmation (gains, losses) affects perceived indirectness. Three studies $(N=589)$ consistently showed that both feedback receivers (Studies 1 and 3) and feedback providers (Study 2) viewed feedback in negation (vs. affirmative) frames as more indirect and communicating the feedback signal (positive, negative) less strongly. Feedback providers utilized more negation frames when attempting to be indirect and delivering failure feedback (Study 2). Further, through influencing perceived positivity in feedback, indirectness has downstream effects on feedback providers' use of frames (Study 2) and feedback receivers' reactions (Study 3). This work contributes to our understanding of communication indirectness and its potential implications for feedback effectiveness.
\end{abstract}

Keywords: Indirectness, feedback, message framing, negation frame, communication 


\section{Message Framing Influences Perceptions of Feedback (In)directness}

Imagine that you have a new assistant to whom you are giving performance feedback. You have not been very impressed, but there is more than one way to convey this feedback. You could tell him directly, "you made several mistakes this month," or you could deliver the news

more subtly, "this is not an easy job for anyone to do." In everyday life, we frequently give and receive feedback - information about how close we are to attaining our goals (Carver \& Scheier, 1982). Feedback communication happens across diverse social roles, both informal (e.g., friends, romantic partners) and formal (e.g., teachers, managers). Effective feedback provides information about goal progress (Locke \& Latham, 1990b) and has the potential to motivate behavior (to continue on the same course or to make changes) (Podsakoff \& Farh, 1989). The extent to which feedback meets these aims depends, in part, on how feedback is conveyed (Kluger \& DeNisi, 1996).

The feedback literature has examined some dimensions on which feedback can differ, influencing its effectiveness factors such as the timeliness and specificity of feedback (Marlett \& Watson, 1968), effort versus ability focus (Dugan, 1989), and task versus process focus (Earley, Northcraft, Lee, \& Lituchy, 1990) influence feedback effectiveness. However, as the opening example illustrates, feedback can also vary in how directly it is communicated. Linguists have long considered indirectness "a fundamental element in human communication” (Tannen, 1994, p. 79). In fact, management practitioners have noted that some leaders express an intention to be indirect when delivering failure feedback, "easing in" to negative messages (e.g., Manzoni, 2002; Meyer, 2014). Yet, the feedback architecture that creates perceptions of indirectness has not been directly studied. Integrating two classic theoretical frameworks - communication indirectness theories (Grice, 1968) and gain/loss message framing (Kahneman \& Tversky, 
1979) — we propose that framing success and failure feedback as an affirmation (gains, losses) versus negation (non-losses, non-gains) will affect how direct versus indirect the feedback is subjectively perceived.

\section{Past Research on Communication Indirectness}

Grice $(1968,1969)$ formalized the idea that a message can be conveyed directly versus indirectly in his early philosophical work on meaning and conversational implicature. According to Grice, any utterance has both a sentence meaning (also called "conventional/timeless meaning”) and a speaker meaning (or “utterer's meaning”). The former refers to the literal meaning of the sentence based on formal definitions of words and rules of sentence structures; the latter refers to the speaker's intentions. According to this original definition, indirectness in a message indicates an incongruence between speaker meaning and sentence meaning, or more broadly, between "any communicative meaning" in the message and the literal meaning of sentences (Holtgraves, 1997a).

Research in the communication literature has suggested multiple ways that speakers can communicate indirectly by inducing meaning incongruence (Grice, 1975). One typical way, most closely tied to Grice's original definition, is by creating a subtle divergence between speaker meaning and sentence meaning (Searle, 1975). For example, if you want someone at the table to pass the salt, you might say, "can you reach the salt?" The literal meaning is whether or not the other person can touch the salt. However, what you intend to communicate is that you want the salt. A second common way in which messages are communicated indirectly is to use hedges or qualifiers to increase the cognitive effort needed to process the literal meaning of a message (Holtgraves, 1994, 1997a). For instance, people often say "Yes, but..." (token agreement) or "I kind of think..." (hedge) before they disagree. These qualifiers, by increasing the complexity of 
the message, soften the direct intention (Holtgraves, 1997b). Although these indirect communication strategies are diverse, they all render speaker meaning harder to interpret, which can sometimes lead to uncertainty and misunderstanding (Hall, 1976).

Indirectness might obscure speaker meaning, but it can also serve crucial social functions. For instance, research has shown that people often use indirectness to be polite and reduce the likelihood of threatening the other person's face (Brown \& Levinson, 1987; Goffman, 1967). At times, a direct request may seem imposing on the listener (e.g., "I want you to pass me the salt"), so the speaker may ask indirectly to convey politeness (e.g., “could you reach the salt?”).

Similarly, directly acknowledging a disagreement between the speaker and listener might cause a listener to lose face. Instead of pointedly calling out the disagreement, the speaker may use a token agreement to lessen the negative impact (e.g., "that is true, but..."). While the motive for politeness has been one of the most studied approaches to understanding why people speak indirectly, it is important to note that indirectness does not equal politeness (see Blum-Kulka, 1987). Indeed, indirectness can serve other very different social functions (e.g., humor, irony; Haverkate, 1990), including some that are arguably the opposite of politeness (e.g., sarcasm). Despite its implications across many interpersonal contexts, indirectness has received scant attention in social psychological work on feedback. The current research addresses this gap and posits that indirectness is likely a particularly relevant factor in understanding the delivery of performance-related feedback, perhaps especially for negative or failure feedback. Failure feedback exchange can be difficult, uncertain, and ego-threatening (Kluger \& DeNisi, 1996). Given that this sometimes leads to the avoidance of giving feedback altogether (Jeffries \& Hornsey, 2012), it may also influence the extent to which feedback providers give feedback directly or indirectly. While feedback could be delivered indirectly in a number of different 
ways, we propose that the message framing literature provides valuable insights into how indirectness in a feedback context may often unfold. Integrating the communication literature with the message framing literature thus not only highlights a new factor that might influence feedback effectiveness, but also suggests a framework to systematically understand communication indirectness itself.

\section{Message Framing and Perceptions of Feedback Indirectness}

Given that no prior work has empirically addressed this phenomenon, we grounded our approach in an eminent and extensive literature examining how subtle differences in languagemessage framing — can have significant consequences. A rich tradition of message framing research has provided evidence that the same objective outcome can be described in terms of gains/non-gains versus non-losses/losses. One of the most prominent examples is provided by Prospect Theory (Kahneman \& Tversky, 1979), which illustrated that subtle differences between a gain and loss frame of the same tangible outcome led to substantial differences in its perceived value.

Since then, understanding people's perceptions toward gain/loss framing is at the center of the message framing literature (Levin, Schneider, \& Gaeth, 1998). Past research has illustrated that gain/loss framing affects perceptions such as riskiness (e.g., Kühberger, 1998; Rothman \& Salovey, 1997), persuasiveness (e.g., Cesario, Higgins, \& Scholer, 2008; Smith \& Petty, 1996), fairness (e.g., Li et al., 2011; Liberman, Idson, \& Higgins, 2005), and choice attractiveness (e.g., Ganzach \& Karsahi, 1995; Lee \& Aaker, 2004). However, no research yet has directly explored the impact of gain/loss framing on perceptions of indirectness. Addressing this gap is essential given that indirectness has many social implications (e.g., Haverkate, 1990; Tannen, 1994), 
including its potential influence on feedback communication (Green \& Carmichael, 2014;

Meyer, 2014).

Affirmative vs. negation frames. Conventionally, the gain/loss framing paradigm varies on two dimensions: message type (i.e., success, failure) and outcome (i.e., gain, loss). As shown in Table 1, success feedback can be framed in terms of gains (presence of positives - "Good work!") or non-losses (absence of negatives - "Not bad!"); failure feedback can be framed in terms of non-gains (absence of positives - "This is not good!") or losses (presence of negatives “This is bad!”) (Cesario, Corker, \& Jelinek, 2013). ${ }^{1}$ Gain- versus loss-framed feedback messages can be used to describe the same objective outcome (Liberman et al., 2005). For instance, a manager who needs to tell her employee that his performance hurt company profits could either tell him that his work led to losses to the company's portfolio (loss frame) or could tell him that his work did not lead to the expected gains (gain frame).

Yet the message and outcome are not the only possible dimensions: Another dimension on which gain/loss framing varies systematically is whether they represent a presence versus an absence of an outcome. We refer to these as 'affirmative' versus 'negation' frames, respectively (see also Yao, Wang, Peng, \& Song, 2017). Affirmative frames are gain-framed successes (gains) and loss-framed failures (losses) — they both represent the presence of positive or negative outcomes. Negation frames are loss-framed successes (non-losses) and gain-framed failures (non-gains) — they both represent the absence of positive or negative outcomes. Building on communication indirectness theories, we propose that the affirmative-versus-negation dimension in message framing has the potential to influence perceptions of indirectness.

\footnotetext{
${ }^{1}$ Note that there have been inconsistencies in past literature in the use of gain and loss frame labels (see Cesario et al., 2013; Smith \& Petty, 1996). Given our discussion of frames intersects with both success and failure, we strictly use the term "gain frames" to refer to either "gain/non-gain" and "loss frames" to refer to either "loss/non-loss".
} 
As Grice (1975) suggested, a message becomes indirect as its literal meaning diverges from its speaker meaning. Consider that success feedback carries a positive speaker meaning and failure feedback carries a negative speaking meaning. By modifying the literal meaning of the feedback, negation (vs. affirmative) frames can create separation between the speaker and literal meaning. Success feedback framed as a negation (non-loss) has a negative frame of reference and may create more meaning separation than an affirmative frame (gain). Similarly, failure feedback framed as a negation (non-gain) has a positive frame of reference and may create more meaning separation than an affirmative frame (loss). Because of the increased separation between the speaker and literal meaning, both success and failure feedback in negation frames, relative to affirmative frames, should be perceived as more indirect — a proposal that has not been empirically tested.

Hypothesis 1 (H1; Studies 1-3): Both feedback receivers and providers will perceive feedback in negation frames (non-losses, non-gains) as more indirect than feedback in affirmative frames (gains, losses).

We will test Hypothesis 1 from the perspective of feedback receivers (Studies 1 and 3) and providers (Study 2). Although we had no a prior reason to expect that the relation between negation frames and indirectness varies as a function of whether individuals are in the role of feedback provider or recipient, it was an empirical question worth exploring. The results of the test of this primary hypothesis have two major theoretical implications. If affirmative and negation frames in feedback affect perceptions of indirectness, the findings will extend the message framing literature by uncovering a novel and critical perception outcome that the 
existing literature has not previously considered. Moreover, while indirectness has been observed in the context of feedback (e.g., Manzoni, 2002; Meyer, 2014), the current work provides a systematic framework for thinking about its architecture. In the following, we propose and test several implications of perceived indirectness as a function of affirmative and negation frames.

Implications for valence perceptions. Indirectness may reduce the clarity of a message (Grice, 1975); in a valenced message such as feedback, indirectness may affect subjective perceptions of feedback valence (i.e., how positive or negative people perceive the feedback to be)_ an important contributor to feedback effectiveness. Some research suggests that negation frames are less hedonically intense than affirmative frames. Monetary outcomes (e.g., a discount, salary) led to greater intensity of positive emotions in gain (affirmative) frames than in non-loss (negation) frames, and greater intensity of negative emotions in loss (affirmative) frames than non-gain (negation) frames (Brendl, Higgins, \& Lemm, 1995; Idson, Liberman, \& Higgins, 2000; Liberman et al., 2005). Based on these observations, framing feedback as a negation versus affirmation may attenuate how intensely people experience the success and failure feedback - their perceptions of feedback valence.

Specifically, success feedback in negation frames (non-losses) relative to affirmative frames (gains) should be perceived as less positive. In contrast, failure feedback in negation frames (non-gains) relative to in affirmative frames (losses), should be perceived as more positive. Moreover, if indeed negation frames attenuate perceptions of valence, it suggests that the difference in perceived valence between success and failure feedback will be weaker in negation (vs. affirmative) frames. This predicted interaction hypothesis involves comparisons of the framing effects on perceived valence both within and between success and failure feedback. 
Hypothesis 2 (H2; Studies 1-3): Perceived valence will be a function of an interaction between frame (negation vs. affirmative) and feedback (success vs. failure):

a) Success feedback in negation (vs. affirmative) frames will be perceived as less positive whereas failure feedback in negation (vs. affirmative) frames will be perceived as more positive (H2a); and

b) The difference in perceived valence between success and failure feedback will be attenuated in negation frames (non-losses vs. non-gains) relative to affirmative frames (gains vs. losses; H2b).

So far, the above hypotheses characterize the vital role of framing feedback in negations versus affirmations in predicting perceptions of feedback indirectness and valence. In the following, we will explore whether indirectness, through influencing perceived valence in feedback, might predict downstream implications for feedback production and reactions.

Implications of indirectness for feedback production. Speakers' intentions matter in speech production (Searle, 1975). In the case of feedback, feedback providers' intentions to be indirect and positive may influence their choice of frames. Feedback providers generally recognize that feedback can be threating to the receiver, especially after a performance failure (Green \& Carmichael, 2014; Kluger \& DeNisi, 1998). To reduce the threat in failure feedback, feedback providers may aim to play up the level of positivity to soften the blow (Manzoni, 2002); being indirect could be one way to do so. In failure feedback, feedback providers may be more likely to use negation (vs. affirmative) frames to the extent that they perceive such frames as reflecting intentions to be indirect and positive. In contrast, success feedback can be 
enhancing to an individual's sense of self (e.g., Heine et al., 2001). Because self-threat is less of a concern, feedback providers may be more likely to use affirmative (vs. negation) frames to the extent that they perceive such frames as reflecting intentions to be direct and positive. In sum, we predict a moderated mediation in which feedback type moderates the relation between intended indirectness and valence, and intended valence, in turn, mediates the relation between intended indirectness and the likelihood of using the frames. We will test this hypothesis in Study 2.

Hypothesis 3 (H3; Study 2): For feedback providers, the relation between intended indirectness and valence will depend on the type of feedback. Negation frames reflect a greater intention to be indirect than affirmative frames: in success feedback, this relative difference will result in affirmative frames reflecting a greater intention to be positive; in failure feedback, the relative difference will result in negation frames reflecting a greater intention to be positive (i.e., moderation). Intentions to be positive, in turn, will prompt a greater likelihood of using the frame (i.e., mediation).

Implications of indirectness for feedback reactions. For feedback receivers, perceptions of the feedback matter; a parallel hypothesis to Hypothesis 3 can also be made. Namely, the relation between perceptions of feedback indirectness and valence may influence more downstream reactions to the feedback. People make observations of a message and infer intentionality of the communicator (see Waytz, Gray, Epley, \& Wegner, 2010). In the case of negative feedback, perceptions of indirectness in the use of negation (vs. affirmative) frames, if associated with perceptions of more positive valence, may indicate that the feedback provider is seeking to minimize the threat. As a result, feedback receivers may view the feedback provider 
as more supportive and report stronger behavioral intentions to commit to future work to the extent that feedback providers use negation versus affirmative frames.

In contrast, in the case of positive feedback, perceptions of directness in the use of affirmative (vs. negation) frames, if associated with perceptions of more positive valence, may indicate that the feedback provider is seeking to bolster the feedback receiver. As a result, feedback receivers may view the feedback provider as more supportive and report stronger behavioral intentions to commit to future work to the extent that feedback providers use affirmative versus negation frames. Integrating these predictions, we hypothesize a moderated mediation in which feedback type moderates the relation between perceptions of indirectness and valence, and perceived valence, in turn, mediates the relations between perceived indirectness and feedback reactions. We will test this hypothesis in Study 3.

Hypothesis 4 (H4; Study 3): For feedback receivers, the relation between perceived indirectness and valence will depend on the type of feedback. Perceived indirectness in failure feedback will be associated with greater perceived positivity, whereas perceived directness in success feedback will be associated with greater perceived positivity (i.e., moderation). Perceived positivity, in turn, will predict i) perceived support from the feedback provider and ii) behavioral intentions to commit to future work (i.e., mediation).

\section{Overview of Studies}

We conducted three experiments to examine our primary prediction (Hypothesis 1) that people perceive negation frames as more indirect than affirmative frames. These studies took the perspective of feedback receivers (Studies 1 and 3) and feedback providers (Study 2), 
generalizing the effects to both sides of a feedback communication. In addition, they examined the effect of feedback framing on perceived valence (Hypothesis 2) and explored the implications of indirectness through positivity on feedback production (Hypothesis 3) and feedback reactions (Hypothesis 4). Demographic variables (e.g., age, gender, ethnicity, and past work experience) did not influence our results and therefore are not included in the analyses below. All measures, manipulations, and exclusions in the study are disclosed, as well as the method of determining the final sample size in the method of each study. All data were analyzed after the completion of data collection, and the sample size of each study was predetermined. These studies provide the first and a rigorous empirical test of how message framing systematically influences communication indirectness.

\section{Study 1 - Feedback Receivers' Perception of Feedback Indirectness}

This study was pre-registered to examine our primary hypothesis that message framing affects perceived indirectness. ${ }^{2}$ Participants imagined a work scenario in which they had succeeded or failed. They then received performance feedback statements that varied in gain and loss frames, and rated their perceptions of each statement (i.e., indirectness and valence). We predicted that people would perceive feedback in negation (vs. affirmative) frames as more indirect (H1), and that negation (vs. affirmative) frames would weaken the detection of the feedback valence $(\mathrm{H} 2)$.

\section{Method}

Participants, design, and power. Participants completed an online study about communication style and personality in a 2(Feedback: Success vs. Failure; between subject) $\times$

\footnotetext{
${ }^{2}$ The pre-registration information of the study is available at osf.io/ma2ez. In real time, we collected the data for this study after all the other studies in this manuscript had been conducted. For coherence and flow of the article, however, we decided to report this study as Study 1.
} 
2(Frame: Negation vs. Affirmative; within-subject) mixed design. We aimed to recruit 200 participants at a large Canadian university who completed the study for psychology course credit. This sample size yields $99 \%$ power to detect a significant Feedback $\times$ Frame interaction with a medium effect size (Faul, Erdfelder, Buchner, \& Lang, 2009). One-hundred and ninetynine undergraduates participated (162 females, 37 males; $M_{\text {age }}=19.8, S D_{\text {age }}=2.2$ ). The sample was culturally diverse (38\% White, 23\% East Asians, 18\% South Asians, and 21\% others). Most participants had some prior work experience (61\%). Participants were first randomly assigned to one of the two feedback conditions. Then they read specific feedback statements that varied in negation and affirmative frames and responded to questions related to each statement. Last, participants completed a battery of other demographic and individual difference questions not related to the current investigation. ${ }^{3}$

Receiving feedback. Participants read a scenario that they had either succeeded or failed in a recent team project at a company (Liden et al., 1999) (see SOM). In the success feedback condition, participants read that:

“...You did particularly well in your assigned task...the overall quality of the project was greatly enhanced, causing unexpectedly high project returns for your team as well as the company..."

In the failure feedback condition, participants read that:

“...You did particularly poorly on your assigned task...the overall quality of the project was severely affected, causing unexpectedly lower project returns for your team as well as the company..."

\footnotetext{
${ }^{3}$ See Supplemental Online Materials for the full list of all exploratory and individual difference measures included in the studies. All analyses reported in the paper were run without any control variables.
} 
Feedback frame manipulation/sentence rating. After reading the scenario, all participants imagined that they received performance feedback from their team leader. The gender of the team leader was matched to the participants' gender. They saw performance feedback statements one at a time and made ratings about perceptions of indirectness and the valence of each statement. Each participant rated 14 statements in random order to avoid any order effects of frames.

In the success condition, participants rated 7 feedback sentences in negation frames (e.g., "Because of your performance, you and your team did not lose the 3 extra paid holidays bonus") and 7 feedback sentences in affirmative frames (e.g., "Because of your performance, you and your team have gained 3 extra paid holidays bonus"). In the failure feedback condition, participants rated 7 feedback sentences in negation frames (e.g., "You would have gained the \$200 project completion bonus if you did not make the mistake") and 7 feedback sentences in affirmative frames (e.g., "you lost the $\$ 200$ project completion bonus due to your mistake") (affirmative frame). All participants rated each sentence in terms of perceived indirectness and valence on a scale from 1 (Very Direct/Very Negative) to 7 (Very Indirect/Very Positive). For each of these variables, the mean score in each feedback frame (i.e., average of 7 sentences) served as the dependent measure in the analysis (Indirectness: $\alpha_{\text {mean }}=.70$; Perceived valence: $\alpha_{\text {mean }}=$.74) (see Appendix for all feedback statements).

\section{Results}

We analyzed ratings of the standardized statements to test Hypotheses 1 and 2. Descriptives and tests of simple effects are reported in Table 2.

Perceived indirectness (H1). A dependent t-test was conducted to compare participants' indirectness ratings of negation and affirmative frames. As predicted, participants perceived 
negation frames as more indirect $(M=3.13, S D=1.18)$ than affirmative frames $(M=2.38$,

$S D=1.07), t(198)=10.93, p<.001, \eta_{\mathrm{p}}{ }^{2}=.38 .^{4}$ Results supported Hypothesis 1.

Perceived valence (H2). We then tested whether perceived valence was a function of an interaction between feedback condition and frame. Ratings of perceived valence of the sentences were analyzed using a 2(Feedback: Success vs. Failure; between-subject $) \times 2$ (Frame: Negation vs. Affirmative; within-subject) repeated-measures ANOVA.

There were significant main effects of Feedback and Frame on perceived valence. Confirming the feedback manipulation, success feedback was perceived as more positive than failure feedback, $F(1,197)=702.27, p<.001, \eta_{\mathrm{p}}{ }^{2}=.78\left(M_{\text {success }}=4.93, S D=.65 ; M_{\text {failure }}=2.48\right.$, $S D=.65$ ). Feedback sentences in negation (vs. affirmative) frames were on average perceived as less positive, $F(1,197)=101.99, p<.001, \eta_{\mathrm{p}}{ }^{2}=.34\left(M_{\text {negation }}=3.43, S D=.78 ; M_{\text {affirmative }}=3.98\right.$, $S D=1.04)$. As predicted, these differences were qualified by a significant Feedback $\times$ Frame interaction, $F(1,197)=475.03, p<.001, \eta_{\mathrm{p}}{ }^{2}=.71$. Specifically, people perceived success feedback in negation (vs. affirmative) frames as less positive, whereas failure feedback in negation (vs. affirmative) frames as more positive (H2a; see Table 2). Moreover, the difference in perceived valence — between success and failure feedback—was attenuated in negation frames, $B=1.25$,

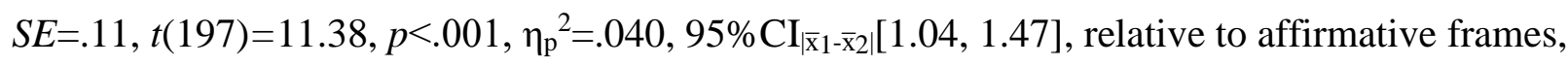
$B=3.64, S E=.10, t(197)=34.89, p<.001, \eta_{\mathrm{p}}{ }^{2}=.86,95 \% \mathrm{CI}_{\left|\bar{x} 1-\bar{x}_{2}\right|}[3.43,3.84](\mathrm{H} 2 \mathrm{~b})$. Results supported Hypothesis 2 that negation (vs. affirmative) frames weaken the detection of the feedback valence.

\footnotetext{
${ }^{4}$ Some frames embedded standardized numerical values (e.g., number of bonus holidays gained or lost, value of profit gained or lost) (6 out of 14 statements), which could arguably be more objectively equivalent than those that did not (8 out of 14 statements). As supplementary analyses, we tested if this matters and results found no supportanalyzing the two types of statements separately yielded the same patterns of perceptions. Therefore, the current finding was unlikely an artifact of varying degrees of information equivalence.
} 


\section{Discussion}

Study 1 provided initial evidence in support of our hypotheses. Feedback frames affected perceptions of indirectness across both success and failure feedback. Negation frames were perceived as less direct than affirmative frames $(\mathrm{H} 1)$ and weakened the detection of the perceived valence (H2). Importantly, negation (vs. affirmative) frames indeed appear to be another approach by which one can create distance between speaker meaning and literal meaning, consistent with Grice's (1975) theories. Building on these results, the next study investigates whether feedback providers' intentions mirror receivers' perceptions observed in Study 1, and moreover, examines whether intentions to be indirect affect their use of frames in providing success and failure feedback.

\section{Study 2 - Feedback Providers' Perception and Use of Frames}

Study 2 takes the feedback providers' perspective and test three hypotheses: whether feedback in negation (vs. affirmative) frames i) reflects a greater intention to be indirect (H1), ii) interacts with feedback type (success vs. failure), to predict intended positivity (H2), and iii) is used to different extents across success and failure feedback because of the dynamics between intended indirectness and positivity (H3). As the focus is on feedback providers, it is meaningful to recruit a sample that has frequent feedback delivery experience. Therefore, we invited fulltime employees who held a supervisory position at work to participate in this study.

Participants played the role of the feedback provider in the scenario from Study 1. In the scenario, they needed to provide feedback to their subordinate who either succeeded or failed. They read standardized negation- and affirmative-framed feedback statements (also adapted from Study 1). For each statement, they responded to three measures: their likelihood of using each frame to provide the feedback, level of intentions to be indirect if they used that frame, and level 
of intentions to be positive if they used that frame. These measures were used to test Hypotheses 1 to 3, and more broadly, allowed the test of whether receivers' perceptions (Study 1) generalize to providers' intentions in feedback communication.

From the feedback providers' perspective, one phenomenon that the standardized statement approach does not capture is the extent to which feedback frames vary naturally in feedback provision. Previously, we hypothesized that feedback providers might recognize that negation (vs. affirmative) frames reflect a greater intention to be indirect (H1). Flipping the relation around, it is also possible that individuals who intend to be more indirect in their feedback may use more negation (vs. affirmative) frames, a phenomenon that is likely to be more pronounced when delivering failure (vs. success) feedback which can be threatening to the recipient (Brown \& Levinson, 1987; Manzoni, 2002).

Our sample of experienced feedback providers offers an opportunity to explore this phenomenon with the help of a behavioral measure. In addition to standardized statements, we asked participants to write performance feedback — as an open-ended response - to the subordinate in the scenario who either succeeded or failed. Afterward, they reported their level of intention to be direct or indirect in the feedback as an individual difference measure. We expected that individual differences in the intention to be indirect might serve as a moderator, enhancing the likelihood of the use of negation frames in failure feedback. To avoid any influence of standardized statements on participants' open-ended responses, participants completed the open-ended response task before reading the standardized statements in the actual study.

\section{Method}


Participants, design, and power. Participants completed an online study about communication style and personality in a 2(Feedback: Success vs. Failure; between-subject) $\times$ 2(Frame: Negation vs. Affirmative; within-subject) mixed design. We aimed to recruit at least 200 participants on Amazon's Mturk, who were U.S. full-time employees and held a supervisory role at work. This sample size yields $99 \%$ power to detect a significant Feedback $\times$ Frame interaction with a medium effect size (Faul et al., 2009). Anticipating some missing data, we recruited 220 people. Among them, 215 participants completed the survey (105 females, 110 males; $M_{\text {age }}=35.2, S D_{\text {age }}=9.87 ; 66 \%$ White, $7 \%$ Black; $6 \%$ Asians, and $21 \%$ others $)$ and most were sales and retail managers and team supervisors. One participant reported not having any former supervisory experience and thus was not included in the analysis. The median number of years of supervisory experience was 6 , and the median number of subordinates was 5 . Five participants had significant missing data and were excluded from the analyses. Another 6 participants provided incomprehensible responses to the open-ended question (e.g., “ewrfdwerf...") and were also excluded. This screening process resulted in a final sample of 203 for analysis. ${ }^{5}$

In this study, participants were randomly assigned to one of two feedback conditions, and then asked to write open-ended feedback to the subordinate. Afterward, they read the feedback sentences that varied in negation and affirmative frames (from Study 1) and responded to questions related to each feedback sentence. Last, they also completed a battery of other demographic and individual differences questions.

Giving feedback. Participants read a scenario in which a gender-matched subordinate performed particularly well or poorly (adopted from Study 1; see SOM). Because we had a work

\footnotetext{
5 The patterns of results were the same with or without excluding participants.
} 
sample, we were also interested knowing whether these scenarios were ecologically valid, resembling real-world situations. At the end of the survey, two questions asked participants whether "situations like that in the scenario happen in your workplace" from 1 (Not at All) to 7 (Very Often) and whether "the scenario appear strange to your experience as a manager/leader" from 1 (Not at All) to 7 (Extremely Strange). Results indicated that these full-time supervisors did experience similar situations at work $(M=4.34, S D=1.48)$, and did not find the scenario to be strange $(M=2.45, S D=1.58)$; there was no difference across conditions, $p s>.190$.

Open-ended feedback writing. Participants wrote open-ended performance feedback to the subordinate. In the success condition, participants were instructed to “...talk to Jesse about his good work and tell him what you feel and what you want him to do..."; in the failure condition, participants were instructed to "...talk to Jesse about his poor work and tell him what you feel and what you want him to do..." (see SOM).

To assess the spontaneous use of frames, three coders independently coded participants' open-ended feedback in random order. Before coding, coders were trained and had to pass a test of accurately differentiating between standard gain/loss framing. These coders individually counted how many times gain and loss frames were used in each participant's feedback writing. Affirmative frames comprise gain-framed success feedback and loss-framed failure feedback, which explicitly mentioned the presence of positive or negative outcomes (e.g., "the project was a great success"); negation frames comprise loss-framed success feedback and gain-framed failure feedback, which explicitly mentioned the absence of positive or negative outcomes (e.g., "you could have put a lot more effort into that project"). Because reliabilities of the coding were high $\left(\alpha_{\text {negation=.89, }}\right.$, and $\left.\alpha_{\text {affirmative }}=.82\right)$, we averaged coders' counts to create an overall score for 
the number of negation and affirmative frames employed in the open-ended feedback for each participant.

Intention to be indirect. Immediately after participants completed their written performance feedback, we measured their conscious intention to be direct or indirect by asking them to indicate how much they were trying to be "as direct as possible" in their feedback (Not at All) to 7 (Very Much). We reverse-coded the rating to reflect participants' intention to be indirect. As we expected that the use of negation (vs. affirmative) frames in feedback depended on the feedback provider's intention to be indirect, this measure served as a moderator in the analysis.

Feedback frame manipulation/sentence rating. Participants were then presented feedback sentences one at a time and made ratings about their i) likelihood to use the frame, ii) intended level of indirectness, and iii) intended level of valence of each sentence. Each participant rated 8 sentences in random order: 4 negation frames and 4 affirmative frames (adopted from Study 1; see SOM). For each sentence, participants were asked the likelihood they would say it (from 1=Very Unlikely to 7=Very Likely), to what extent they would be trying to be indirect if they said it (from 1=Very Direct to 7=Very Indirect), and to what extent they would be trying to be positive if they said it (from 1=Very Negative to 7=Very Positive). Each of the three variables formed two mean scores, one for negation frames and one for affirmative frames, which served as the dependent measures in the analysis (Indirectness: $\alpha_{\text {mean }}=.67$; Valence: $\alpha_{\text {mean }}=.69$; Likelihood to say: $\alpha_{\text {mean }}=.63$ ).

\section{Results}


Following the order of the hypotheses, we first reported results of the ratings of the standardized statements, and then moved on to analyzing the open-ended feedback. See Table 2 for descriptives and tests of simple effects.

\section{Standardized feedback frames.}

Intended indirectness (H1). A dependent t-test was conducted to compare participants' indirectness ratings of negation and affirmative frames. As predicted, participants viewed negation frames as reflecting a greater intention to be indirect $(M=3.06, S D=1.13)$ than affirmative frames $(M=3.06, S D=1.13), t(202)=9.99, p<.001, \eta_{\mathrm{p}}{ }^{2}=.33$ (see Figure 1). This finding replicated Study 1, providing evidence that feedback providers also recognize negation (vs. affirmative) frames as more indirect.

Intended valence (H2). Next, we tested whether intended valence was a function of an interaction between feedback condition and frame. Ratings of the intended valence of the sentences were analyzed using a 2 (Feedback: Success vs. Failure; between-subject) $\times 2$ (Frame: Negation vs. Affirmative; within-subject) repeated-measures ANOVA.

There was a significant main effect of Feedback, $F(1,201)=285.59, p<.001, \eta_{\mathrm{p}}{ }^{2}=.59$ $\left(M_{\text {success }}=5.06, S D=.92 ; M_{\text {failure }}=2.87, S D=1.16\right)$, and a significant main effect of Frame, $F(1$, $202)=88.31, p<.001, \eta_{\mathrm{p}}{ }^{2}=.31\left(M_{\text {negation }}=3.62, S D=1.08 ; M_{\text {affirmative }}=4.31, S D=1.04\right)$. Importantly, as predicted in $\mathrm{H} 2$, the main effects were qualified by a significant Feedback $\times$ Frame interaction, $F(1,201)=424.96, p<.001, \eta_{\mathrm{p}}{ }^{2}=.68$. Success feedback in negation (vs. affirmative) frames was viewed as reflecting a weaker intention to be positive, whereas failure feedback in negation (vs. affirmative) frames was viewed as reflecting a stronger intention to be positive (H2a; see Table 2). Moreover, the difference in intended levels of valence-between success and failure feedback - was attenuated in negation frames, $B=.65, S E=.15, t(201)=4.29, p<.001$, 
$\eta_{\mathrm{p}}{ }^{2}=.08,95 \% \mathrm{CI}_{|\overline{\mathrm{x}} 1-\overline{\mathrm{x}} 2|}[.35, .95]$, relative to affirmative frames, $B=3.71, S E=.15, t(201)=25.50$, $p<.001, \eta_{\mathrm{p}}^{2}=.76,95 \% \mathrm{CI}_{\left|\overline{\mathrm{x}}_{1}-\overline{\mathrm{x}}_{2}\right|}[3.43,4.00](\mathrm{H} 2 \mathrm{~b})$.

Moderated mediation (H3). We then examined whether the likelihood of using a frame was influenced by the intentions to be indirect and positive in a moderated mediation model. We hypothesized that, in success feedback, affirmative frames reflect a greater intention to be direct and positive; in failure feedback, negation frames reflect a greater intention to be indirect and positive (i.e., moderation). Intended positivity of frames, in turn, will prompt a greater likelihood of using the frame (i.e., mediation).

To test the moderated mediation, we followed the percentile bootstrapping approach of moderated mediation with 10,000 bootstrapped samples (Preacher, Rucker, \& Hayes, 2007; Model 8). Because of the within-subjects design of Frame, we first created difference scores (negation frame scores minus affirmative frame scores) within each of the variables: indirectness, valence, and likelihood to use the frame. Second, we included the grand meancentered means (across negation and affirmative frames) of indirectness and valence as controls (see Montoya \& Hayes, 2017). ${ }^{6}$ This analysis included a regression test for the interaction effect between intended indirectness and feedback condition on intended valence, along with a test for the mediating effect of intended valence on the likelihood to use the frame.

As Figure 3 indicates, the intention to be indirect interacted with feedback condition, predicting feedback providers' intended valence, $p=.001,95 \% \mathrm{CI}[-.40,-.10]$. Intended valence, in turn, predicted the likelihood of using the frame in feedback, $p<.001,95 \% \mathrm{CI}[.53, .77]$. The indirect effects of indirectness on the likelihood to use the frame were asymmetrical: negative for successful feedback, but positive for failure feedback. This means that, to make feedback more

\footnotetext{
${ }^{6}$ Without the controls yielded a similar pattern and significance level of the indirect effects.
} 
positive, feedback providers intended to deliver failure (vs. success) feedback using frames that they considered more (vs. less) indirect. A test of moderated mediation index was significant, $B=-.33, S E=.11,95 \% \mathrm{CI}[-.58,-.14]$, suggesting that the two indirect effects differed from each other and intended indirectness predicts divergent usage of frame in success and failure feedback. Supplementary analyses switching the order of the variables in the mediation model rendered both indirect effects and moderated mediation indices nonsignificant, providing additional support for the predicted direction of the indirect effects.

Coding of open-ended feedback (H1). Last, we turned to examine participants' openended feedback (completed prior to rating the sentences above). We conducted a repeated measure ANOVA, using the coders' negation and affirmative frame scores as the within-subject dependent variables. As we expected that the use of frames would depend on the individual difference in feedback providers' intention, we examined participants' intention to be indirect as a moderator, along with the frame and feedback condition.

There was a significant main effect of Frame, such that participants on average used more affirmative than negation frames, $F(1,202)=102.18, p<.001, \eta_{\mathrm{p}}{ }^{2}=.34\left(M_{\text {negation }}=1.02, S D=1.21\right.$; $\left.M_{\text {affirmative }}=2.80, S D=1.69\right)$. There were no main effects of Feedback, $F(1,201)=1.52, p=.219$, and of the intention to be indirect, $F(1,201)=.91, p=.342$. Importantly, we expected that individuals who intend to be more indirect in their feedback may use more negation (vs. affirmative) frames (i.e., an alternative form of H1). Supporting this notion, there was a significant Frame $\times$ Intention to be Indirect interaction, $F(1,201)=12.23, p=.001, \eta_{\mathrm{p}}{ }^{2}=.06$. Indeed, feedback providers' intention to be indirect was associated with the use of more negation frames, $t(202)=2.98$, $p=.003, \eta_{\mathrm{p}}{ }^{2}=.04$, and less affirmative frames, $t(202)=-3.02, p=.003, \eta_{\mathrm{p}}{ }^{2}=.04$. It is noteworthy that this interaction was qualified by a higher-order three-way interaction between Feedback $\times$ Frame 
$\times$ Intention to be Indirect, $F(1,199)=9.55, p=.002, \eta_{\mathrm{p}}{ }^{2}=.05$, suggesting that the relation between the intention to be indirect and use of frame depended on the type of feedback (see Figure 3 ). ${ }^{7}$ For failure feedback, the Frame $\times$ Intention to be Indirect interaction was significant, $F(1$, $98)=10.05, p=.002, \eta_{\mathrm{p}}{ }^{2}=.09$. The stronger the participant's intention to be indirect when writing the failure feedback, the more negation frames they used, $B=.24, S E=.10, p=.017,95 \% \mathrm{CI}[.04$, $.44]$, and the fewer affirmative frames they used, $B=-.23, S E=.11, p=.036,95 \% \mathrm{CI}[-.44,-.02]$. For success feedback, there was no significant interaction between Frame and Intention to be Indirect, $F(1,101)=1.47, p=.228, \eta_{\mathrm{p}}{ }^{2}=.01$. Taken together, results suggested that individuals who intend to be more indirect in their feedback would use more negation (vs. affirmative) frames (H1), yet this pattern was driven primarily by the delivery of feedback on a performance failure, not a success.

\section{Discussion}

In sum, Study 2 showed that feedback providers' intentions mirrored feedback receivers' perceptions (Study 1). Results of standardized feedback sentences supported Hypotheses 1 to 3. Feedback providers viewed negation (vs. affirmative) frames as reflecting a greater intention to be indirect (H1). Feedback frames interacted with feedback type, success and failure, to predict intended positivity (H2). Feedback providers reported a greater likelihood of using negation (vs. affirmative) frames for failure (vs. success) feedback to the extent that intended indirectness was associated with intended positivity $(\mathrm{H} 3)$. In a replication study using a comparable design but an

\footnotetext{
7 . The Feedback $\times$ Intention to be Indirect interaction was nonsignificant, $F(1,199)=1.02, p=.314 .2$. The Feedback $\times$ Frame interaction was significant, $F(1,201)=248.17, p<.001, \eta_{\mathrm{p}}{ }^{2}=.55$. Participants used more affirmative frames to deliver success (vs. failure) feedback, $B=2.08, S E=.19, t=11.12, p<.001, \eta_{\mathrm{p}}{ }^{2}=.38,95 \% \mathrm{CI}[1.71$, 2.45], and more negation frames to deliver failure (vs. success) feedback, $B=-1.81, S E=.11, t=-16.02, p<.001$, $\eta_{\mathrm{p}}{ }^{2}=.56,95 \% \mathrm{CI}[-2.04,-1.59]$. For success feedback, participants wrote more affirmative frames than negated loss frames, $t(102)=25.17, p<.001, \eta_{\mathrm{p}}{ }^{2}=.86,95 \% \mathrm{CI}[-3.99,-3.41]$. For failure feedback, participants used similar numbers of negated and affirmative frames, $t(99)=1.15, p=.251, \eta_{\mathrm{p}}{ }^{2}=.01$.
} 
undergraduate sample $(N=196)$, we found a very similar pattern of results (see supplementary study details in SOM). Thus, these results appear robust and do not depend on age or work experience.

Moreover, Study 2 included a measure of spontaneous feedback provision behavior that has higher external validity compared to standardized sentence ratings. Analyzing participants' spontaneous feedback revealed that the individual difference in the intention to be indirect matters for the use of frame. Feedback providers' intention to be indirect was associated with a greater use of negation (vs. affirmative) frames. This tendency primarily happened in failure feedback, consistent with prior work emphasizing the importance of indirectness in selfthreatening and face-sensitive situations (Brown \& Levinson, 1987; Holtgraves, 1997b). Together, Study 2 results suggest that feedback providers understand the contribution of negation frames to indirectness, and take advantage of negation frames in feedback provision when they intend to be indirect.

\section{Study 3 - Feedback Receivers' Perceptions Revisited}

Study 2 showed that feedback providers indeed regulated indirectness in feedback communication, influencing how the feedback was framed. The mediation analyses suggest that this stems, at least in part, from an intention to be positive. Study 3 builds on these findings by returning to the perspective of the feedback receiver. Going beyond the results that indirectness (vs. directness) in failure (vs. success) feedback felt more positive, Study 3 investigated downstream consequences.

As discussed in the introduction, it is possible that perceived indirectness, through influencing perceived positivity, affect reactions to the feedback. Particularly, we hypothesized that, in failure feedback perceived indirectness will be associated with greater perceived 
positivity, whereas in success feedback perceived directness will be associated with greater perceived positivity (i.e., moderation). Perceived positivity, in turn, will predict i) perceived support from the feedback provider and ii) behavioral intentions to commit to future work (i.e., mediation; H4). As in Study 2, these relations will be examined using a moderated mediation analysis.

Last, we made a methodological change to enhance the impact of this study. Instead of asking participants to rate individual feedback statements, this study manipulated feedback in the form of an email. This provides a more naturalistic context for feedback provision and serves as a conceptual replication of previous findings. That is, we used a different experimental paradigm to test whether people perceive feedback in negation (vs. affirmative) frames as more indirect (H1), and whether negation (vs. affirmative) frames weaken the detection of the feedback valence $(\mathrm{H} 2)$.

\section{Method}

Participants, design, and power. Participants participated in an online survey about communication style and personality in a 2 (Feedback: Success vs. Failure) $\times 2$ (Frame: Negation vs. Affirmative) between-subject design. We aimed to recruit approximately 200 participants over an academic term for psychology course credit; this would yield $94 \%$ power to detect a Frame $\times$ Feedback interaction with a medium effect size. One hundred and ninety-eight undergraduates at a large Canadian university participated ( 151 females, 45 males; $M_{\text {age }}=20.8$, $S D_{\text {age }}=5.6 ; 56 \%$ White, $17 \%$ East Asians, $11 \%$ South Asians, 5\% Southeast Asians and $11 \%$ others; $65 \%$ have work experience). Among these participants, 11 had missing data and were excluded from the analysis, resulting in a final sample of 187 people. In this study, participants first completed a battery of demographic and other individual difference questions. They were 
then randomly assigned to one of the two feedback conditions, asked to imagine the work scenario and read feedback in an email from their supervisor. Afterward, they responded to questions related to the feedback.

Receiving feedback. Similar to Study 1, participants read a gender-matched scenario in which they were told they had performed well (success) or poorly (failure) on a recent team project at a company (Liden et al., 1999) (see SOM).

Feedback framing manipulation in the email. Participants then imagined that they received an email (negation- vs. affirmative-framed) from their team project leader about their performance. For consistency, these emails were composed of the feedback statements in negation and affirmative frames adapted from Study 2 (see the emails in SOM). After reading, participants rated the email in terms of perceived indirectness and positivity on a scale from 1 (Very Direct/Very Negative) to 7 (Very Indirect/Very Positive).

Perceived support. Based on the scenario, we included 3 items to measure participants' perception of support from the feedback provider (Amabile, Schatzel, Moneta, \& Kramer, 2004; $\alpha=.86$ ); for example, "I receive encouragement and support from the team leader" from 1 (Not at all) to 7 (Extremely).

Behavioral intentions. We measured post-feedback behavioral intentions to work with a 12-item scale adapted from Lockwood, Jordan, and Kunda (2002); $\alpha=.85$. A sample item was, "I plan to work harder for the coming project" from 1 (Not at all) to 7 (Extremely).

\section{Results and Discussion}

We first tested Hypotheses 1 and 2 with ratings of the feedback email, and then moved on to analyzing feedback reactions. See Table 2 for descriptives and tests of simple effects. 
Perceived indirectness (H1). An independent t-test was conducted to investigate whether perceptions of indirectness differed across feedback emails with negation versus affirmative frames. As predicted, participants perceived the emails with negation frames as more indirect $(M=2.49, S D=1.63)$ than the emails with affirmative frames $(M=1.93, S D=1.47)$, $t(185)=2.46, p=.015, \eta_{\mathrm{p}}{ }^{2}=.03$ (see Figure 1). The result is consistent with Studies 1 and 2 findings and supported Hypothesis 1.

Perceived valence (H2). We then conducted a 2(Feedback: Positive vs. Negative) $\times$ 2(Frame: Negation vs. Affirmative) between-subject ANOVA to analyze perceived valence of the emails. There were significant main effects of Feedback, $F(1,183)=112.80, p<.001, \eta_{\mathrm{p}}{ }^{2}=.38$ $\left(M_{\text {success }}=4.57, S D=1.32 ; M_{\text {failure }}=2.52, S D=1.33\right)$ and Frame on perceived valence of the email, $F(1,183)=17.50, p<.001, \eta_{\mathrm{p}}{ }^{2}=.09\left(M_{\text {negation }}=3.14, S D=1.30 ; M_{\text {affirmative }}=3.95, S D=1.35\right)$. As predicted, the Feedback $\times$ Frame interaction was significant, $F(1,183)=17.50, p<.001, \eta_{\mathrm{p}}{ }^{2}=.09$. The success feedback email in negation (vs. affirmative) frames was perceived as less positive, whereas the failure feedback email in negation (vs. affirmative) frames was intended to be more positive (H2a; see Table 2). For emails using affirmative frames, perceived valence between success and failure feedback differed significantly, $F(1,88)=260.04, p<.001, \eta_{\mathrm{p}}{ }^{2}=.75$. However, for emails using negation frames, perceived valence between success and failure feedback did not differ, $F(1,95)=1.71, p=.194, \eta_{\mathrm{p}}^{2}=.02(\mathrm{H} 2 \mathrm{~b})$. Results supported Hypothesis 2 .

\section{Behavioral intentions and perceived support as downstream consequences (H4).}

Next, we examined feedback reactions as the downstream consequences of perceived indirectness. Specifically, we tested whether the asymmetrical effects of indirectness across success and failure feedback would be related to perceived support from the feedback provider and behavioral intentions. 
Perceived support. We used the same bootstrapping approach of moderated mediation in Study 2, except we did not employ difference scores because of the between-subject design. As Figure 4 indicates, perceived indirectness interacted with feedback condition in predicting perceived valence of the feedback, $p=.004,95 \% \mathrm{CI}[-.43,-.08]$. The more people perceived the email as positive, the more they perceived the feedback provider as supportive, $p<.001$, 95\% CI[.48, .71]. As expected, the indirect effects of perceived indirectness were asymmetrical. Through increased positivity of the email, more direct success feedback led to more perceived support; in contrast, more indirect failure feedback tended to result in more perceived support. The test of moderated mediation index showed that the two conditional indirect effects were independent as they differed significantly from each other, $B=-.31, S E=.11,95 \% \mathrm{CI}[-.56,-.10]$.

Behavioral intentions. We also tested the conditional indirect effects of indirectness on behavioral intentions. As seen in Figure 4, the more people perceived the email as positive the more they reported stronger work-relevant behavioral intentions, $p<.001,95 \% \mathrm{CI}[.09, .32] .{ }^{8}$ The indirect effects of perceived indirectness on behavioral intentions were again asymmetrical. Through increased positivity of the email, more direct success feedback led to stronger behavioral intention to work harder; in contrast, more indirect failure feedback led to stronger behavioral intention to work harder. The test of moderated mediation index revealed that the two conditional indirect effects differed significantly from each other, $B=-.10, S E=.05,95 \% \mathrm{CI}[-.21$, .03]. ${ }^{9}$ Results provided support for Hypothesis 4.

\section{General Discussion}

\footnotetext{
${ }^{8}$ A Feedback $\times$ Frame interaction (instead of the Feedback $\times$ Indirectness interaction) produced the same pattern of results. For the sake of consistency with earlier studies, we reported the model using the Feedback $\times$ Indirectness interaction.

${ }^{9}$ Supplementary analyses switching the order of the variables in the mediation model of perceived support and of behavioral intentions rendered both insignificant indirect effects and moderated mediation indices, providing additional support for these current and specific directions of the indirect effects.
} 
Three experiments examined how feedback in negation vs. affirmative frames affected perceived indirectness. Both feedback receivers (Studies 1 and 3) and feedback providers (Study 2) perceived success and failure feedback in negation frames (non-losses, non-gains) as more indirect than in affirmative frames (gains, losses; Hypothesis 1). Success feedback in negation (vs. affirmative) frames was perceived as less positive whereas failure feedback in negation (vs. affirmative) frames was perceived as more positive. Hence, the difference in perceived valence between success and failure feedback was attenuated (Hypothesis 2). Results were consistent whether feedback frames were manipulated in individual feedback statements (Studies 1 and 2) or an integrated email (Study 3). Further, feedback providers (e.g., full-time supervisors; Study 2) appeared to recognize these dynamics and were more likely to use negation frames when they intended to be indirect in giving feedback. Taken together, these studies consistently demonstrate the effect of message framing on the perception of indirectness in feedback communications.

\section{The Dynamics of Indirectness in Providing Effective Feedback}

The current work integrates the communication and message framing literatures to provide new insights into the role of indirectness in feedback communication. Although prior work has examined the role of other factors — timeliness, specificity, task/person focus—on feedback, this is the first empirical work to examine factors that contribute to perceived indirectness. As Grice's $(1968,1969)$ theories suggested, to the extent that a speaker meaning is distant from the literal meaning, communication becomes more indirect. The present studies reveal one form of this distance in the context of feedback provision: framing a success as a nonloss or a failure as a non-gain.

Moreover, the current studies suggest that feedback indirectness may play an interesting role in feedback provision and receipt, affecting the clarity of the feedback valence signal and 
interpersonal dynamics between feedback provider and receiver. This is important as feedback communication is a core component of many social roles. For instance, a major task of a manager is to give feedback effectively, and ineffective feedback can lead to many severe organizational downfalls (e.g., lower motivation and resentment; Baron, 1988). Ineffective feedback provided by teachers can lower students' learning and academic achievement (Rattan, Good, \& Dweck, 2012). Our findings add to the growing feedback literature by highlighting the dynamic role of indirectness in feedback effectiveness.

In the case of providing success feedback, the story of indirectness creates no great challenges or surprises: being direct is related to more accurate detection of the feedback signal, perceptions of greater support from the feedback provider, and strengthened behavioral intentions for future work. Interestingly, however, people may be more accustomed both to receiving success feedback and to directness when they do, so the same dose of directness in success feedback may not feel as strong as the same dose of directness in negative feedback (see Baumeister, Bratslavsky, Finkenauer, \& Vohs, 2001). This might explain why a pattern emerged that people perceived success (vs. failure) feedback as more indirect overall (see Figure 1). In the case of failure feedback, however, the current studies suggest that feedback providers might face a dilemma in how direct to be.

Feedback receivers perceived indirect failure feedback — in negation frames — as more positive than direct failure feedback - in affirmative frames. On the one hand, this appears to have some benefits, as revealed in Study 3. Feedback recipients felt more supported when they received indirect failure feedback, one source of goal (and certainly work) engagement (Amabile et al., 2004) and they reported stronger behavioral intentions for future work. On the other hand, feedback providers may be less able to pick up the valence signal when failure feedback is 
indirect. Indeed, receivers did not differentiate the valence of success versus failure feedback when it was in negation frames in the form of an email (Study 3), even though objective performance anchors were explicitly provided in the scenario. Thus, the attempt to "soften the blow" by giving failure feedback indirectly may make harder for receivers to recognize that something has gone wrong and/or needs to be addressed.

A critical function of feedback is to give people information about goal discrepancies (Locke \& Latham, 1990a). Realizing that one's performance is discrepant from the desired state often leads to greater goal engagement in order to reduce the discrepancy (Carver \& Scheier, 1982; Carver, 2003). Thus, although directness in failure feedback may make an individual feel worse at the moment, it may also make the discrepancy toward the goal more salient, triggering increased motivation to adjust future behavior. In Study 3, we assessed behavioral intentions to work harder in general, rather than assessing behavioral intentions to fix problematic behaviors/past mistakes. It is possible that indirect negative feedback makes it less likely for individuals to take these corrective steps. Thus, we believe it will be important for future work to consider how indirectness of failure feedback affects more distal motivation and behavior, given likely tensions between directness and indirectness in negative feedback.

\section{Exploration of Potential Mechanisms and Moderators}

Although we began to explore why feedback providers might deliver feedback in affirmative versus negation frames in Study 2, we recognize that there is much more to understand about the potential mechanisms of this effect. Feedback providers indicated that to the extent they were trying to be indirect in feedback provision, they were more likely to use negation (non-gain) frames for failure feedback. While this is consistent with past work suggesting that indirectness often serves the goal of being polite (Brown \& Levinson, 1987; 
Goffman, 1967), there may be additional motives at play in the context of feedback provision. For example, indirect failure feedback might be perceived not only as being more positive for receivers, but also as creating a more comfortable (and face-saving) interpersonal exchange for the feedback provider. Thus, feedback providers might have both receiver-relevant and selfrelevant motives for delivering feedback indirectly.

Similarly, it will also be interesting to explore if the effects of indirect feedback are the same for everyone. For example, people vary in their chronic levels of communication indirectness: they can be more or less indirect in their production and/or interpretation of communication (Holtgraves, 1997a). People who are chronically more indirect in speech production may be more likely to use negation frames when communicating feedback.

There may also be self-regulatory factors that influence what is experienced as motivating, such as described in regulatory focus theory (Higgins, 1997). Regulatory focus differentiates between two critical self-regulatory systems - promotion and prevention - that regulate different survival needs. In a promotion state, individuals are concerned with nurturance and advancement, are particularly sensitive to the difference between gains and non-gains. In contrast, in a prevention state, individuals are concerned with safety and responsibilities, are particularly sensitive to the difference between non-losses and losses. Past work has shown that gain frames are more motivating for promotion-focused individuals whereas loss-frames are more motivating for prevention-focused individuals (e.g., Kung, Kim, Yang, \& Cheng, 2016). Together this suggests that regulatory focus might be an important moderating factor that drives both how feedback providers frame feedback and how feedback receivers are motivated (or not) by specific feedback frames.

\section{Limitations and Recommendations for Future Research}


Before we conclude, we draw attention to several limitations of the current work. All studies in the current work employed hypothetical scenarios. Despite many benefits of using such an experimental design, this approach may have limited mundane realism. Results may not fully reflect the psychological experience when people receive real performance feedback or when people's performance outcome has high stakes (e.g., affecting actual profits). Future research should aim to test the hypotheses in real-world performance situations, such as performance appraisal in companies and academic evaluation at schools, to increase the external validity of the research.

Additionally, building on the message framing literature, we began our work with a focus on variations in gain/loss framing as a critical antecedent of perceived indirectness. Yet, other antecedents are also essential and future research should explore alternative means by which indirectness can be created. For example, a separation between speaker and literal meaning may also be created by a discrepancy between verbal utterances and nonverbal behaviors, such as facial expressions, emotional tones, and social distance (LaPlante \& Ambady, 2002, 2003). For instance, if your colleague says, "I am thrilled about working on this task" while expressing no positive affect, it is unlikely that this is your colleague's favorite job. Future research will benefit from exploring the variety of means people use to create indirectness in communication and their implications.

Last, our study samples were all recruited in North America, and we do not have direct evidence to show whether the results can generalize across national cultures. Cultural influences are critical in communication. For example, cultures can vary in the degree to which communication tends to be direct versus indirect (Hall, 1976). Hall differentiated between highcontext cultures (e.g., Russia, China) that use more situational and nonverbal cues to 
communicate meaning versus low-context cultures (e.g., Canada, Germany). These cultural norms may influence both the antecedents and consequences of indirect feedback (Meyer, 2014), as well as influence beliefs about what is motivating. Therefore, future research should increase the diversity of national cultures in their sample and continue to explore these intriguing questions.

\section{Conclusion}

Indirectness is important, but underexplored, in feedback provision. The present studies showed that subtle variations in gain/loss framing that produce negation vs. affirmative frames influence the extent to which success and failure are perceived as more or less direct. Particularly in the case of failure, the implications of negation frames are complex, affecting detection of the valence signal and motivation in divergent ways. We believe that further explorations of the role of message framing and indirectness in feedback communications will ultimately lead to a deeper understanding of when and why social interactions go well or awry. 


\section{References}

Amabile, T. T. M., Schatzel, E. A. E., Moneta, G. G. B., \& Kramer, S. J. (2004). Leader behaviors and the work environment for creativity: Perceived leader support. The Leadership Quarterly, 15(1), 5-32. http://doi.org/10.1016/j.leaqua.2003.12.003

Baron, R. A. (1988). Negative effects of destructive criticism: Impact on conflict, self-efficacy, and task performance. Journal of Applied Psychology, 73(2), 199-207. http://doi.org/10.1037/0021-9010.73.2.199

Baumeister, R. F., Bratslavsky, E., Finkenauer, C., \& Vohs, K. D. (2001). Bad is stronger than good. Review of General Psychology, 5(4), 323-370. http://doi.org/10.1037/10892680.5.4.323

Blum-Kulka, S. (1987). Indirectness and politeness in requests: Same or different? Journal of Pragmatics, 11(2), 131-146. http://doi.org/10.1016/0378-2166(87)90192-5

Brendl, C. M., Higgins, E. T., \& Lemm, K. M. (1995). Sensitivity to varying gains and losses: The role of self- discrepancies and event framing. Journal of Personality and Social Psychology, 69(6), 1028-1051. http://doi.org/10.1037/0022-3514.69.6.1028

Brown, P., \& Levinson, S. (1987). Politeness: Some universals in language usage. Cambridge, UK: Cambridge University Press.

Carver, C. S. (2003). Pleasure as a sign you can attend to something else: Placing positive feelings within a general model of affect. Cognition \& Emotion, 17(2), 241-261.

Carver, C. S., \& Scheier, M. F. (1982). Control theory: A useful conceptual framwork for personality-social, clinical, and health psychology. Psychological Bulletin, 92(1), 111-135.

Cesario, J., Corker, K. S., \& Jelinek, S. (2013). A Self-regulatory framework for message framing. Journal of Experimental Social Psychology, 49, 238-249. 
http://doi.org/10.1016/j.jesp.2012.10.014

Cesario, J., Higgins, E. T., \& Scholer, A. A. (2008). Regulatory fit and persuasion: Basic principles and remaining questions. Social and Personality Psychology Compass, 2(1), 444-463. http://doi.org/10.1111/j.1751-9004.2007.00055.x

Dugan, K. W. (1989). Ability and effort attributions: Do they affect how managers communicate performance feedback information? Academy of Management Journal, 32(1), 87-114. http://doi.org/10.2307/256421

Earley, P. C., Northcraft, G. B., Lee, C., \& Lituchy, T. R. (1990). Impact of process and outcome feedback on the relation of goal setting to task performance. Academy of Management Journal, 33(1), 87-105. http://doi.org/10.2307/256353

Faul, F., Erdfelder, E., Buchner, A., \& Lang, A.-G. (2009). Statistical power analyses using G*Power 3.1: tests for correlation and regression analyses. Behavior Research Methods, 41(4), 1149-1160. http://doi.org/10.3758/BRM.41.4.1149

Ganzach, Y., \& Karsahi, N. (1995). Message framing and buying behavior: A field experiment. Journal of Business Research, 32(1), 11-17. http://doi.org/10.1016/0148-2963(93)00038-3

Goffman, E. (1967). Interaction ritual: essays on face-to-face interaction. Oxford, England: Aldine.

Green, S., \& Carmichael, S. G. (2014, June). Everything You Need to Know About Giving Negative Feedback. Harvard Business Review. Retrieved from https://hbr.org/2014/06/everything-you-need-to-know-about-negative-feedback

Grice, H. P. (1968). Utterer's meaning, sentence-meaning, and word-meaning. Foundations of Language, 4(3), 225-242.

Grice, H. P. (1969). Utterer's meaning and intention. The Philosophical Review, 78(2), 147-177. 
http://doi.org/10.2307/2184179

Grice, H. P. (1975). Logic and conversation. In P. Cole \& J. Morgan (Eds.), Syntax and semantics 3: Speech arts (pp. 41-58). New York: Academic Press.

Hall, T. E. (1976). Beyond Culture. Garden City, NY: Anchor.

Haverkate, H. (1990). A speech act analysis of irony. Journal of Pragmatics, 14(1), 77-109. http://doi.org/10.1016/0378-2166(90)90065-L

Heine, S. J., Kitayama, S., Lehman, D. R., Takata, T., Ide, E., Leung, C., \& Matsumoto, H. (2001). Divergent consequences of success and failure in Japan and North America: An investigation of self-improving motivations and malleable selves. Journal of Personality and Social Psychology, 81(4), 599-615. http://doi.org/10.1037/0022-3514.81.4.599

Higgins, E. T. (1997). Beyond pleasure and pain. American Psychologist, 52(12), 1280-1300. http://doi.org/10.1037/0003-066X.52.12.1280

Holtgraves, T. (1994). Communication in context: Effects of speaker status on the comprehension of indirect requests. Journal of Experimental Psychology: Learning, Memory, and Cognition, 20(5), 1205-1218. http://doi.org/10.1037/0278-7393.20.5.1205

Holtgraves, T. (1997a). Styles of language use: Individual and cultural variability in conversational indirectness. Journal of Personality and Social Psychology, 73(3), 624-637. http://doi.org/10.1037/0022-3514.73.3.624

Holtgraves, T. (1997b). Yes, but... Positive politeness in conversation arguments. Journal of Language and Social Psychology, 16(2), 222-239.

Idson, L. C., Liberman, N., \& Higgins, E. T. T. (2000). Distinguishing gains from nonlosses and losses from nongains: a regulatory focus perspective on hedonic intensity. Journal of Experimental Social Psychology, 36(3), 252-274. http://doi.org/10.1006/jesp.1999.1402 
Jeffries, C. H., \& Hornsey, M. J. (2012). Withholding negative feedback: Is it about protecting the self or protecting others? British Journal of Social Psychology, 51(4), 772-780. http://doi.org/10.1111/j.2044-8309.2012.02098.x

Kahneman, D., \& Tversky, A. (1979). Prospect theory: An analysis of decision under risk. Econometrica: Journal of the Econometric Society, 47(2), 263-291.

Kluger, A. N., \& DeNisi, A. (1996). The effects of feedback interventions on performance: A historical review, a meta-analysis, and a preliminary feedback intervention theory. Psychological Bulletin, 119(2), 254-284. http://doi.org/10.1037/0033-2909.119.2.254

Kluger, A. N., \& DeNisi, A. (1998). Feedback interventions: Toward the understanding of a double-edged sword. Current Directions in Psychological Science, 7(3), 67-72. http://doi.org/10.2307/20182507

Kühberger, A. (1998). The influence of framing on risky decisions: A meta-analysis. Organizational Behavior and Human Decision Processes, 75(1), 23-55. http://doi.org/10.1006/obhd.1998.2781

Kung, F. Y. H., Kim, Y.-H., Yang, D. Y. J., \& Cheng, S. Y. Y. (2016). The role of regulatory fit in framing effective negative feedback across cultures. Journal of Cross-Cultural Psychology, 47(5), 696-712. http://doi.org/10.1177/0022022116638172

LaPlante, D., \& Ambady, N. (2002). Saying it like it isn't: Mixed messages from men and women in the workplace. Journal of Applied Social Psychology, 32(12), 2435-2457. http://doi.org/10.1111/j.1559-1816.2002.tb02750.x

LaPlante, D., \& Ambady, N. (2003). On how things are said - Voice tone, voice intensity, verbal content, and perceptions of politeness. Journal of Language and Social Psychology, 22(4), 434-441. http://doi.org/10.1177/0261927x03258084 
Lee, A. Y., \& Aaker, J. L. (2004). Bringing the frame into focus: the influence of regulatory fit on processing fluency and persuasion. Journal of Personality and Social Psychology, 86(2), 205-218. http://doi.org/10.1037/0022-3514.86.2.205

Levin, I. P., Schneider, S. L., \& Gaeth, G. J. (1998). All frames are not created equal: A typology and critical analysis of framing effects. Organizational Behavior and Human Decision Processes, 76(2), 149-188. http://doi.org/10.1006/obhd.1998.2804

Li, A., Evans, J., Christian, M. S., Gilliland, S. W., Kausel, E. E., \& Stein, J. H. (2011). The effects of managerial regulatory fit priming on reactions to explanations. Organizational Behavior and Human Decision Processes, 115(2), 268-282.

http://doi.org/10.1016/j.obhdp.2011.01.003

Liberman, N., Idson, L. C., \& Higgins, E. T. (2005). Predicting the intensity of losses vs. nongains and non-losses vs. gains in judging fairness and value: A test of the loss aversion explanation. Journal of Experimental Social Psychology, 41(5), 527-534. http://doi.org/10.1016/j.jesp.2004.06.007

Liden, R. C., Wayne, S. J., Judge, T. A., Sparrowe, R. T., Kraimer, M. L., \& Franz, T. M. (1999). Management of poor performance: A comparison of manager, group member, and group disciplinary decisions. Journal of Applied Psychology, 84(6), 835-850. http://doi.org/10.1037/0021-9010.84.6.835

Locke, E. A., \& Latham, G. P. (1990a). A theory of goal setting \& task performance. Englewood Cliffs, NJ, US: Prentice-Hall, Inc.

Locke, E. A., \& Latham, G. P. (1990b). Work motivation and satisfaction: light at the end of the tunnel. Psychological Science, 1(4), 240-246. http://doi.org/10.1111/j.14679280.1990.tb00207.x 
Lockwood, P., Jordan, C. H., \& Kunda, Z. (2002). Motivation by positive or negative role models: Regulatory focus determines who will best inspire us. Journal of Personality and Social Psychology, 83(4), 854-864. http://doi.org/10.1037/0022-3514.83.4.854

Manzoni, J.-F. (2002, September). A Better Way to Deliver Bad News. Harvard Business Review. Retrieved from https://hbr.org/2002/09/a-better-way-to-deliver-bad-news

Marlett, N. J., \& Watson, D. (1968). Test anxiety and immediate or delayed feedback in a testlike avoidance task. Journal of Personality and Social Psychology, 8(2), 200-203. http://doi.org/10.1037/h0025273

Meyer, E. (2014). How To Say “This Is Crap” In Different Cultures. Harvard Business Review. Retrieved from https://hbr.org/2014/02/how-to-say-this-is-crap-in-different-cultures

Montoya, A. K., \& Hayes, A. F. (2017). Two-condition within-participant statistical mediation analysis: A path-analytic framework. Psychological Methods, 22(1), 6-27. http://doi.org/10.1037/met0000086

Podsakoff, P. M., \& Farh, J.-L. (1989). Effects of feedback sign and credibility on goal setting and task performance. Organizational Behavior and Human Decision Processes, 44(1), 4567. http://doi.org/10.1016/0749-5978(89)90034-4

Preacher, K. J., Rucker, D. D., \& Hayes, A. F. (2007). Addressing moderated mediation hypotheses: Theory, methods, and prescriptions. Multivariate Behavioral Research, 42(1), 185-227. http://doi.org/10.1080/00273170701341316

Rattan, A., Good, C., \& Dweck, C. S. (2012). "It's ok — Not everyone can be good at math": Instructors with an entity theory comfort (and demotivate) students. Journal of Experimental Social Psychology, 48(3), 731-737. http://doi.org/10.1016/j.jesp.2011.12.012 Rothman, A. J., \& Salovey, P. (1997). Shaping perceptions to motivate healthy behavior: The 
role of message framing. Psychological Bulletin, 121(1), 3-19. http://doi.org/10.1037/00332909.121.1.3

Searle, J. R. (1975). Indirect speech acts. In P. Cole \& J. Morgan (Eds.), Syntax and semantics 3: Speech arts (pp. 59-82). New York: Academic Press.

Smith, S. M., \& Petty, R. E. (1996). Message framing and persuasion: A message processing analysis. Personality and Social Psychology Bulletin, 22(3), 257-268. http://doi.org/10.1177/0146167296223004

Tannen, D. (1994). Talking from 9 to 5: Women and men at work. New York: William Morrow.

Waytz, A., Gray, K., Epley, N., \& Wegner, D. M. (2010). Causes and consequences of mind perception. Trends in Cognitive Sciences, 14(8), 383-388.

http://doi.org/10.1016/j.tics.2010.05.006

Yao, S., Wang, Y., Peng, J., \& Song, L. (2017). The framing effect of negation frames. Journal of Risk Research, 1-9. http://doi.org/10.1080/13669877.2017.1281335 
Table 1. Negation and Affirmative Feedback Frames

\begin{tabular}{lcc}
\cline { 2 - 3 } $\begin{array}{l}\text { Outcome } \\
\text { frame }\end{array}$ & \multicolumn{2}{c}{ Feedback } \\
\cline { 2 - 3 } Gain Frame & $\begin{array}{c}\text { Gain } \\
\text { (Affirmative) } \\
\text { Loss Frame }\end{array}$ & $\begin{array}{c}\text { Non-gain } \\
\text { (Negation) } \\
\text { Nons }\end{array}$ \\
\hline
\end{tabular}


Table 2. Descriptives, Tests of Simple Effects, and Comparisons Across Dependent Variables in All Studies.

\begin{tabular}{|c|c|c|c|c|c|c|c|c|}
\hline & & \multicolumn{2}{|c|}{ Means (SD) } & $d f$ & $F$ & $p$ & $\eta_{\mathrm{p}}^{2}$ & $95 \% \mathrm{CI}_{\mid \overline{\mid \overline{1} 1-\bar{x} 2}}$ \\
\hline & & & \multicolumn{6}{|c|}{ Study 1} \\
\hline \multirow{3}{*}{ Indirectness } & & Negation & Affirmative & & & & & \\
\hline & Success & $3.69(1.06)^{\mathrm{a}}$ & $2.67(1.03)^{\mathrm{b}}$ & 98 & 85.40 & $<.001$ & .47 & $.77,1.19$ \\
\hline & Failure & $2.57(.98)^{\mathrm{c}}$ & $2.10(1.03)^{\mathrm{d}}$ & 99 & 55.82 & $<.001$ & .36 & $.35, .60$ \\
\hline \multirow[t]{2}{*}{ Valence } & Success & $4.13(1.06)^{b}$ & $5.80(.69)^{\mathrm{a}}$ & 98 & 330.53 & $<.001$ & .77 & $1.48,1.85$ \\
\hline & Failure & $2.80(.76)^{\mathrm{c}}$ & $2.19(1.03)^{\mathrm{d}}$ & 99 & 125.35 & $<.001$ & .56 & $.50, .72$ \\
\hline
\end{tabular}

\section{Study 2}

\begin{tabular}{llccccccc}
\multirow{2}{*}{ Indirectness } & Success & $3.87(1.22)^{\mathrm{a}}$ & $2.62(1.33)^{\mathrm{b}}$ & 102 & 75.37 & $<.001$ & .42 & $.97,1.54$ \\
& Failure & $2.65(1.17)^{\mathrm{b}}$ & $2.18(1.29)^{\mathrm{c}}$ & 99 & 37.12 & $<.001$ & .27 & $.32, .62$ \\
\multirow{2}{*}{ Valence } & & & & & & & & \\
& Success & $3.94(1.14)^{\mathrm{b}}$ & $6.17(.71)^{\mathrm{a}}$ & 102 & 360.55 & $<.001$ & .78 & $2.00,2.46$ \\
& Failure & $3.29(1.02)^{\mathrm{c}}$ & $2.46(1.29)^{\mathrm{d}}$ & 99 & 85.62 & $<.001$ & .46 & $.65,1.01$
\end{tabular}

\section{Study 3}

\begin{tabular}{llccccccc}
\multirow{2}{*}{ Indirectness } & Negation & Affirmative & & & & & \\
& Success & $3.13(1.83)^{\mathrm{a}}$ & $2.38(1.73)^{\mathrm{b}}$ & 92 & 4.13 & .045 & .04 & $.02,1.47$ \\
& Failure & $1.90(1.16)^{\mathrm{b}}$ & $1.44(.93)^{\mathrm{c}}$ & 91 & 4.28 & .041 & .05 & $.02, .90$ \\
Valence & Success & $3.34(1.52)^{\mathrm{b}}$ & $5.81(1.14)^{\mathrm{a}}$ & 92 & 79.42 & $<.001$ & .46 & $1.92,3.02$ \\
& Failure & $2.94(1.49)^{\mathrm{b}}$ & $2.09(1.04)^{\mathrm{c}}$ & 91 & 9.77 & .002 & .10 & $.31,1.39$
\end{tabular}

Note. Within each perception variable, means that share the same letter superscript are not statistically different from each other $(p>.05)$. Studies 1 and 2 have a mixed design of Frame (within-subject) $\times$ Feedback (between-subject). Study 3 has a $2 \times 2$ between-subject design . $95 \% \mathrm{CI}\left|\overline{\mathrm{x}}_{1}-\overline{\mathrm{x}}_{2}\right|$ represents the $95 \%$ confidence interval of the absolute difference in means. 


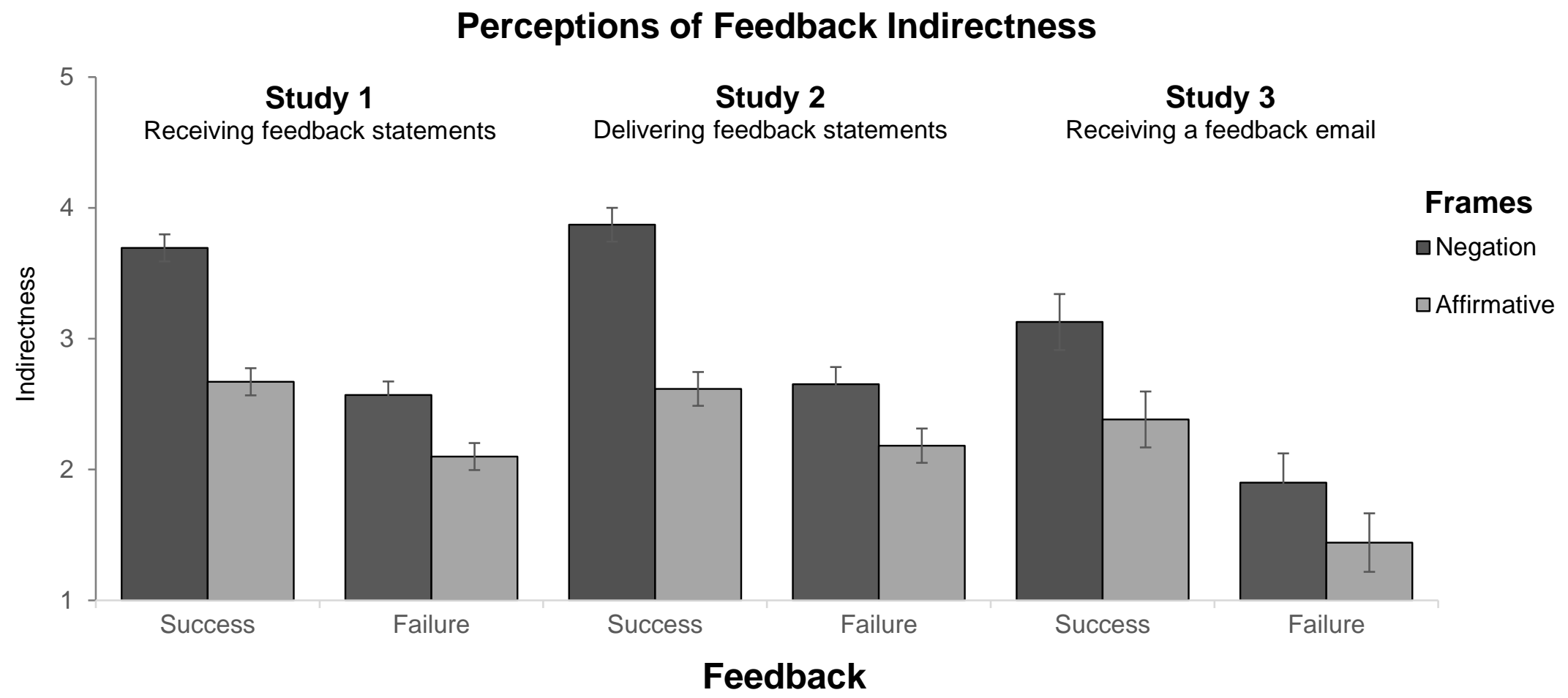

Figure 1. A Panel of Perceived Indirectness of Feedback in Negation and Affirmative Frames across Success and Failure Feedback Conditions in All Studies. Error bars are means +/- 1 S.E (within-conditions). 


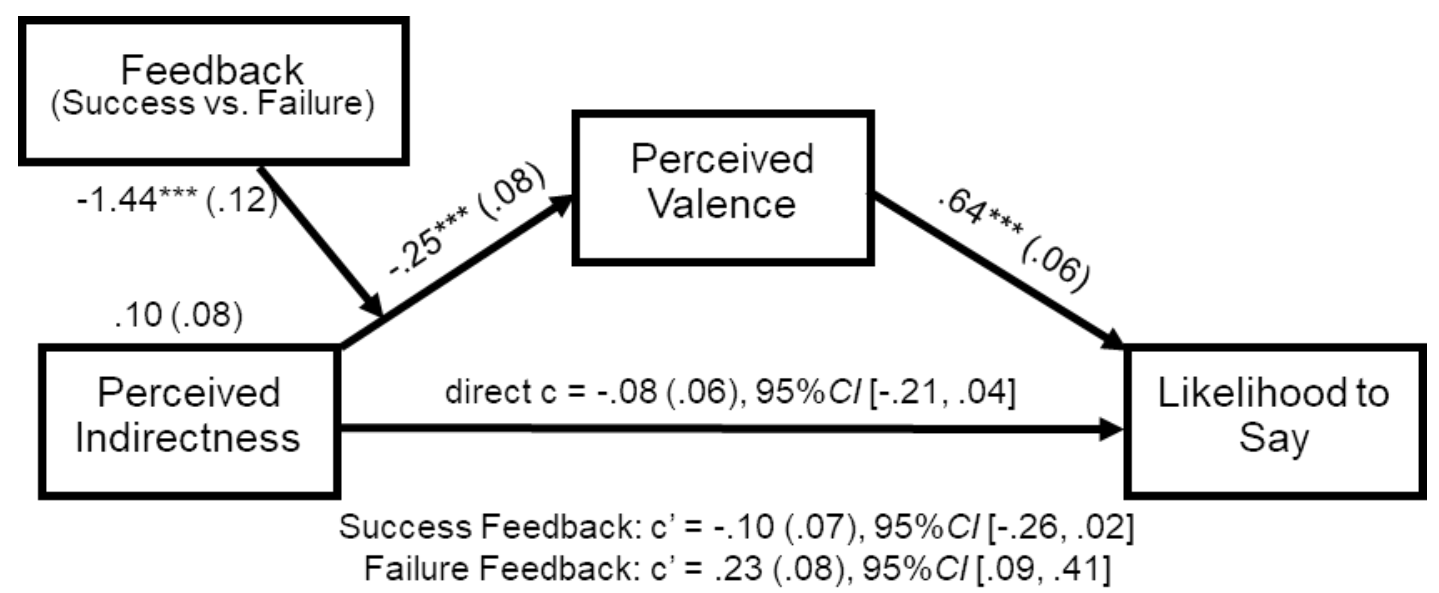

Figure 2. The Indirect Effect of Perceived Indirectness on the Likelihood to Use the Frame via Perceived Valence, Moderated by Feedback Condition (Study 2). Estimates are unstandardized coefficients with standard errors reported in parentheses (see all estimates in Table S3). Feedback conditions: Success $=1$ and Failure $=-1$. Perceived indirectness was mean-centered in the analysis. $* * * p \leq .001$. 


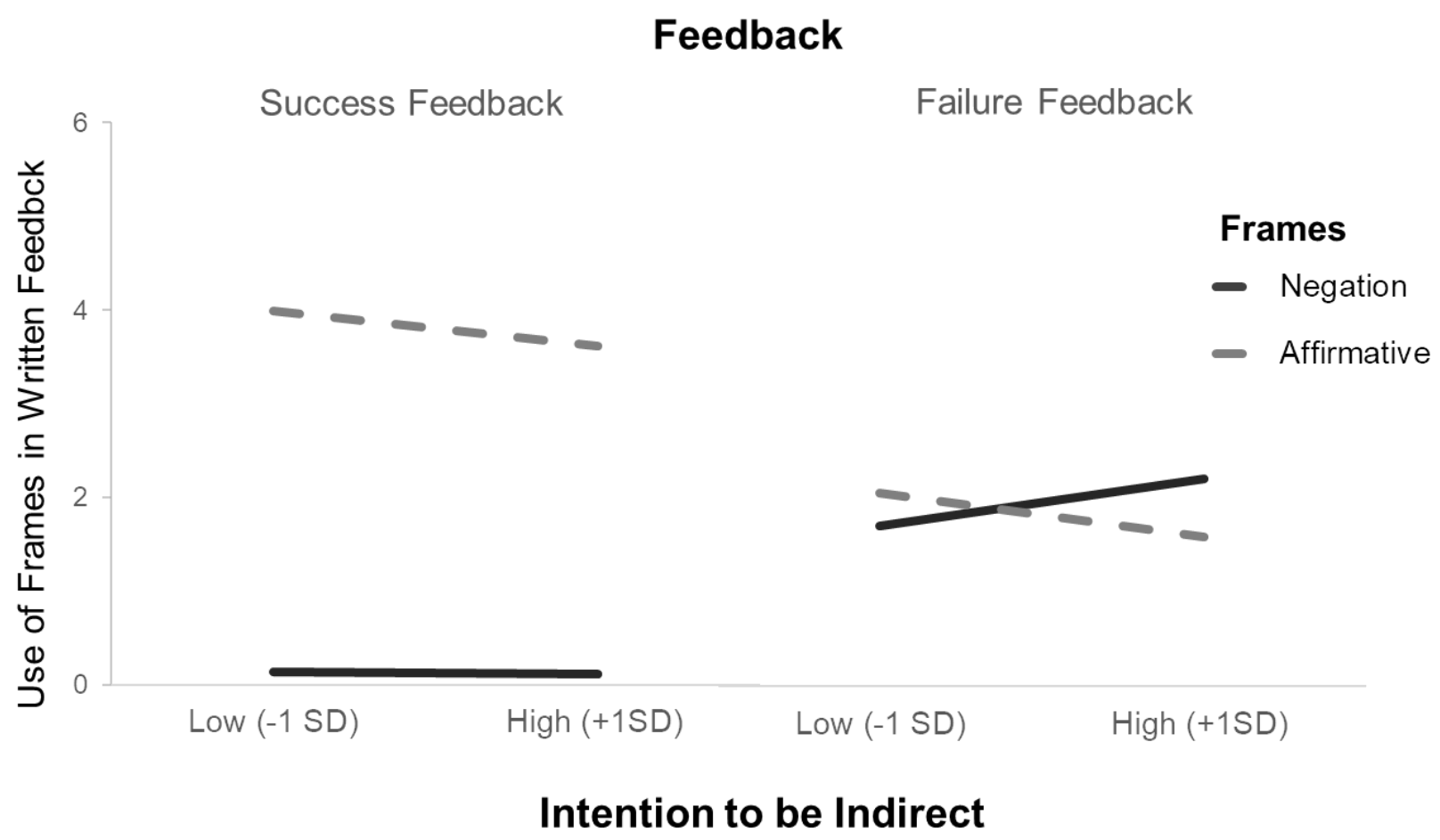

Figure 3. Number of Negation and Affirmative Frames Used as a Function of Feedback Providers' Intention to be Indirect and Feedback Conditions (Study 2). 


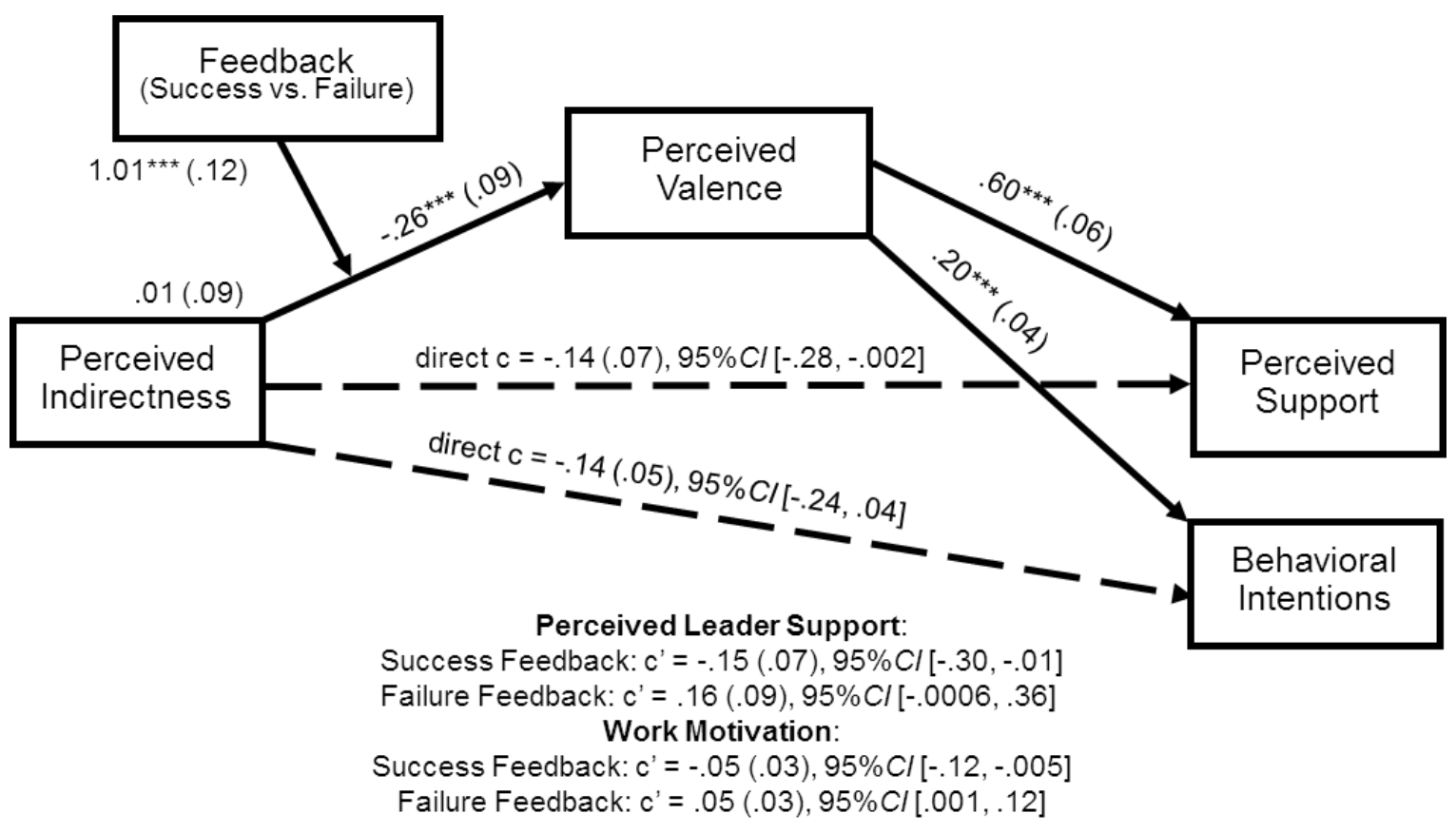

Figure 4. The Indirect Effect of Perceived Indirectness of the Feedback Email on Perceived Support and Behavioral Intentions via Perceived Valence, Moderated by Feedback Condition (Study 3). Estimates are unstandardized coefficients with standard errors reported in parentheses (see all estimates in Table S4). Feedback conditions: Success $=1$ and Failure $=-1$. Perceived indirectness was mean-centered in the analysis. $* * * p \leq .001$. 


\section{Appendix: Feedback Statements in Negation and Affirmative Frames}

Feedback

\begin{tabular}{|c|c|c|}
\hline Frame & Success & $\begin{array}{c}\text { Failure } \\
\end{array}$ \\
\hline \multirow[t]{7}{*}{ Affirmative } & $\begin{array}{l}\text { In the part for which you were } \\
\text { responsible, we have achieved the } \\
\text { ideal gain. } 1,2, \mathrm{~S}\end{array}$ & $\begin{array}{l}\text { In the part for which you were } \\
\text { responsible, we lost money. } 1,2, \mathrm{~S}\end{array}$ \\
\hline & $\begin{array}{l}\text { Your performance in the project was } \\
\text { strong. } 1,2, \mathrm{~S}\end{array}$ & $\begin{array}{l}\text { Your performance in the project was } \\
\text { poor. } 1,2, S\end{array}$ \\
\hline & You did very well in the task. ${ }^{1,2, \mathrm{~S}}$ & You did quite badly in the task. $1,2, \mathrm{~S}$ \\
\hline & $\begin{array}{l}\text { Our team achieved the ideal amount of } \\
\text { profits because of your outstanding } \\
\text { work. }{ }^{1,2, S}\end{array}$ & $\begin{array}{l}\text { We lost money because of your } \\
\text { mistakes. } 1,2, \mathrm{~S}\end{array}$ \\
\hline & $\begin{array}{l}\text { Because of your performance, you and } \\
\text { your team have gained } 3 \text { extra paid } \\
\text { holidays bonus. }{ }^{1}\end{array}$ & $\begin{array}{l}\text { Because of your performance, you and } \\
\text { your team lost } 2 \text { paid holidays bonus. }\end{array}$ \\
\hline & $\begin{array}{l}\text { Due to your performance, you have } \\
\text { gained } \$ 200 \text { extra toward your project } \\
\text { completion bonus. }{ }^{1}\end{array}$ & $\begin{array}{l}\text { You lost the } \$ 200 \text { project completion } \\
\text { bonus due to your mistake. }{ }^{1}\end{array}$ \\
\hline & $\begin{array}{l}\text { The company made } \$ 12,000 \text { as profit, } \\
\text { out of which } \$ 2,000 \text { was extra } \\
\text { investment we gained because of your } \\
\text { performance. }^{1}\end{array}$ & $\begin{array}{l}\text { The company had a chance to gain } \\
\$ 10,000 \text { as profit. However, owing to } \\
\text { your performance, we achieved } \$ 8000 \text {, } \\
\text { losing } \$ 2,000 \text { in profit. }{ }^{1}\end{array}$ \\
\hline \multirow[t]{7}{*}{ Negation } & $\begin{array}{l}\text { In the part for which you were } \\
\text { responsible, we did not suffer from } \\
\text { any losses. } 1,2, \mathrm{~S}\end{array}$ & $\begin{array}{l}\text { In the part for which you were } \\
\text { responsible, we didn't make gains. } 1,2, \mathrm{~S}\end{array}$ \\
\hline & $\begin{array}{l}\text { Your performance in the project was } \\
\text { not bad. } 1,2, \mathrm{~S}\end{array}$ & $\begin{array}{l}\text { Your performance in the project was } \\
\text { not strong. } 1,2, \mathrm{~S}\end{array}$ \\
\hline & You did not fail in the task. ${ }^{1,2, \mathrm{~S}}$ & $\begin{array}{l}\text { You could have done better in the } \\
\text { task. } 1,2, \mathrm{~S}\end{array}$ \\
\hline & $\begin{array}{l}\text { Our team would have lost some } \\
\text { money but for your outstanding } \\
\text { work. } 1,2, \mathrm{~S}\end{array}$ & $\begin{array}{l}\text { We could have gotten more profits if it } \\
\text { were not for your mistakes. } 1,2, \mathrm{~s}\end{array}$ \\
\hline & $\begin{array}{l}\text { Because of your performance, you and } \\
\text { your team did not lose the } 3 \text { extra paid } \\
\text { holidays bonus. }{ }^{1}\end{array}$ & $\begin{array}{l}\text { Because of your performance, you and } \\
\text { your team did not gain the } 2 \text { paid } \\
\text { holidays bonus. }{ }^{1}\end{array}$ \\
\hline & $\begin{array}{l}\text { Due to your performance, you did not } \\
\text { lose the } \$ 200 \text { extra toward your } \\
\text { project completion bonus. }\end{array}$ & $\begin{array}{l}\text { You would have gained the } \$ 200 \\
\text { project completion bonus, if you did } \\
\text { not make the mistake. }\end{array}$ \\
\hline & $\begin{array}{l}\text { The company made } \$ 12,000 \text { as profit, } \\
\text { out of which } \$ 2,000 \text { was extra } \\
\text { investment we would have lost if not } \\
\text { for your performance. }{ }^{1}\end{array}$ & $\begin{array}{l}\text { The company had a chance to gain } \\
\$ 10,000 \text { as profit. However, owing to } \\
\text { your performance, we achieved } \$ 8000 \text {, } \\
\text { missing out on gaining } \$ 2000 \text { in } \\
\text { profit. }\end{array}$ \\
\hline
\end{tabular}

Note. ${ }^{1}$ Included in Study $1 ;{ }^{2}$ Included in Study 2; Included in the Supplementary Study. 